\title{
A Space-Saving Approximation Algorithm for Grammar-Based Compression
}

\author{
Hiroshi SAKAMOTO $^{\dagger a)}$, Member, Shirou MARUYAMA ${ }^{\dagger \dagger b)}$, Takuya KIDA ${ }^{\dagger \dagger \dagger c)}$, \\ and Shinichi SHIMOZONO ${ }^{\dagger \mathrm{d})}$, Nonmembers
}

\begin{abstract}
SUMMARY A space-efficient approximation algorithm for the grammar-based compression problem, which requests for a given string to find a smallest context-free grammar deriving the string, is presented. For the input length $n$ and an optimum CFG size $g$, the algorithm consumes only $O(g \log g)$ space and $O\left(n \log ^{*} n\right)$ time to achieve $O\left(\left(\log ^{*} n\right) \log n\right)$ approximation ratio to the optimum compression, where $\log ^{*} n$ is the maximum number of $\log$ arithms satisfying $\log \log \cdots \log n>1$. This ratio is thus regarded to almost $O(\log n)$, which is the currently best approximation ratio. While $g$ depends on the string, it is known that $g=\Omega(\log n)$ and $g=O\left(\frac{n}{\log _{k} n}\right)$ for strings from $k$-letter alphabet [12].

key words: grammar-based compression, approximation algorithm, minimum CFG problem
\end{abstract}

\section{Introduction}

The grammar-based compression problem is to find a smallest context-free grammar generating just single string. Such a CFG requires that every nonterminal is derived from only one production rule, say, deterministic. The problem deeply relates to factoring problems for strings, and the complexity of similar minimization problems have been rigorously studied. For example, Storer [20] introduced a factorization for a given string and showed the problem is NP-hard. De Agostino and Storer [2] defined several online variants and proved that those are also NP-hard.

As non-approximability results, Lehman and Shelat [13] showed that the problem is APX-hard, i.e. it is hard to approximate this problem within a constant factor (see [1] for definitions). They also mentioned its interesting connection to the semi-numerical problem [9], which is an algebraic problem of minimizing the number of different multiplications to compute the given integers and has no known polynomial-time approximation algorithm achieving a ratio $o(\log n / \log \log n)$. Since the problem is a special case of the

\footnotetext{
Manuscript received March 28, 2008.

Manuscript revised June 19, 2008.

†The authors are with the Faculty of Computer Science and Systems Engineering, Kyushu Institute of Technology, Iizuka-shi, 820-8502 Japan.

${ }^{\dagger}$ The author is with the Department of Informatics, Kyushu University, Fukuoka-shi, 819-0395 Japan.

${ }^{\dagger \dagger \dagger}$ The author is with the Graduate School of Information Science and Technology, Hokkaido University, Sapporo-shi, 0600814 Japan.

a)E-mail: hiroshi@ai.kyutech.ac.jp

b)E-mail: shiro.maruyama@i.kyushu-u.ac.jp

c) E-mail: kida@ist.hokudai.ac.jp

d) E-mail: sin@ai.kyutech.ac.jp

DOI: 10.1587/transinf.E92.D.158
}

grammar-based compression, an approximation better than this ratio seems to be also hard.

On the other hand, various practical algorithms for the grammar-based compression have been devised so far. LZW [21] including LZ78 [24], and BISECTION [8] are considered as algorithms that computes straight-line programs, CFGs formed from Chomsky normal form formulas. Also algorithms for restricted CFGs have been presented in [6], [10], [15], [16], [22]. Lehman and Shelat [13] proved the upper bounds of the approximation ratio of these practical algorithms, as well as the lower bounds with the worst-case instances. For example, BISECTION algorithm achieves an approximation ratio no more than $O\left((n / \log n)^{1 / 2}\right)$. All those ratios, including the lower-bounds, are larger than $O(\log n)$.

Recently polynomial-time approximation algorithms for the grammar-based compression problem have been widely studied and the worst-case approximation ratio has been improved. The first $\log n$-approximation algorithm was developed by Charikar et al. [4]. Their algorithm guarantees the ratio $O(\log (n / g))$, where $g$ is the size of a minimum deterministic CFG for an input. Independently, Rytter presented in [17] another $O(\log (n / g))$-approximation algorithm that employs a suffix tree and the LZ-factorization technique for strings. Sakamoto also proposed in [19] a simple lineartime algorithm based on Re-pair [10] and achieving ratio $O(\log n)$; Now this ratio has been improved to $O(\log (n / g))$.

The ratio $O(\log (n / g))$ achieved by these new algorithms is theoretically sufficiently small. However, all these algorithms require $O(n)$ space, and it prevents us to apply the algorithms to huge texts, which is crucial to obtain a good compression ratio in practice. For example, the algorithm Re-pair [10] spends $5 n+n^{1 / 2}$ space on unit-cost RAM with the input size $n$.

This state motivates us to develop a sub-linear space $O(\log n)$-approximation algorithm for the grammar-based compression. We presented a simple algorithm [18] that repeats substituting one new nonterminal symbol to all the same and non-overlapping two contiguous symbols occurring in the string. This is carried out by utilizing idea of the lowest common ancestor of balanced binary trees, and no real special data structure, such as suffix tree or occurrence frequency table, is requested. In consequence, the space complexity is nearly equal to the total number of created nonterminal symbols, each of which corresponds to a production rule in Chomsky normal form. This algorithm was applied to Compressed Pattern Matching in [14]. In this 
paper we improve the algorithm and obtain almost $O(\log n)$ approximation ratio preserving the space complexity.

The size of the final dictionary of the rules is proved by the compactness of LZ-factorization [17] and alphabet reduction technique [5]. This technique requires $\log ^{*} n$ times iteration. Here $\log ^{*} n$ denotes the maximum integer $j$ which satisfies $F(j) \leq n$ for

$$
F(0)=1 \text {, and } F(j)=2^{F(j-1)}(j \geq 1) .
$$

For instance, $F(3)=2^{4}=16, F(4)=2^{16}=65536$, and $F(5)=2^{65536}$. Thus, $\log ^{*} n$ is almost constant even for sufficiently large $n$. Our algorithm runs in almost $O(n)$ time and $O(g \log g)$ space preserving the worst-case approximation ratio $O\left(\left(\log ^{*} n\right) \log n\right)$. This ratio is almost the currently best approximation. The memory space is devoted to the dictionary that maps a contiguous pair of symbols to a nonterminal. Practically, in randomized model, space complexity can be reduced to $O(g \log g)$ by using a hash table for the dictionary. In the framework of dictionary-based compression, the lower-bound of memory space is usually estimated by the size of a possible smallest dictionary, and thus our algorithm is nearly optimal in space complexity. Compared to other practical dictionary-based compression algorithms, such as LZ78, which achieves the ratio $\Omega\left(n^{2 / 3} / \log n\right)$, the lower-bound of memory space of our algorithm is considered to be sufficiently small.

The remaining part of this paper is organized as follows. In Sect. 2, we prepare the definitions related to the grammar-based compression. In Sect. 3, we introduce the notion of lowest common ancestors in a complete binary tree defined by alphabet symbols. Using this notion, our algorithm decides a fixed priority of all pairs appearing in a current string and replaces them according to the priority. More precisely, a pair is called to be maximal if its priority is higher than the neighbors'. The aim of the algorithm is to find as many maximal pairs as possible, and this is performed by iterative application of the alphabet reduction. The algorithm is presented in Sect. 4 and we analyze the approximation ratio and estimate the time/space efficiency compared with related grammar-based compression algorithms. In Sect. 5, we summarize this study.

\section{Notions and Definitions}

In this study we suppose a standard RAM model [11] with the unit-cost measure, in which the following assumptions are made. Each value is a primitive data item, the memory required by a given variable is equal to the number of entries in the array that it represents, the memory required by a RAM is equal to the total memory required by its variables, and the time required by a RAM is equal to the number of instructions being executed.

We next recall the notions in formal language theory. Given a sufficiently large integer $n$ for the input length, we assume that the size of any symbol is bounded by $O(\log n)$ bits, and a finite set $\Sigma$ of symbols is called an alphabet. The set of all strings over $\Sigma$ is denoted by $\Sigma^{*}$, and
$\Sigma^{i}$ denotes the set of all strings of length just $i$. The length of a string $w \in \Sigma^{*}$ is denoted by $|w|$, and also for a set $S$, the notion $|S|$ refers to the size (cardinality) of $S$. The $i$ th symbol of $w$ is denoted by $w[i]$. For an interval $[i, j]$ with $1 \leq i \leq j \leq|w|$, the occurrence of a substring from $w[i]$ to $w[j]$ is denoted by $w[i, j]$.

A repetition is a string $x^{k}$ for some $x \in \Sigma$ and some positive integer $k$. A repetition $w[i, j]$ in $w$ of a symbol $x \in \Sigma$ is maximal if $w[i-1] \neq x$ and $w[j+1] \neq x$. It is simply referred by $x^{+}$if there is no ambiguity in its interval in $w$. Intervals $[i, j]$ and $\left[i^{\prime}, j^{\prime}\right]$ with $i<i^{\prime}$ are overlapping if $i^{\prime} \leq$ $j<j^{\prime}$, and are independent if $j<i^{\prime}$. A pair $u \in \Sigma^{2}$ is a string of length two, and an interval $[i, i+1]$ is a segment of $u$ in $w$ if $w[i, i+1]=u$.

A context-free grammar $(C F G)$ is a quadruple $G=$ $(\Sigma, N, P, s)$ of disjoint finite alphabets $\Sigma$ and $N$, a finite set $P \subseteq N \times(N \cup \Sigma)^{*}$ of production rules, and the start symbol $s \in N$. Symbols in $N$ are called nonterminals. A production rule $a \rightarrow b_{1} \cdots b_{k}$ in $P$ derives $\beta \in(\Sigma \cup N)^{*}$ from $\alpha \in(\Sigma \cup N)^{*}$ by replacing an occurrence of $a \in N$ in $\alpha$ with $b_{1} \cdots b_{k}$. In this paper, we assume that any CFG is deterministic, that is, for each nonterminal $a \in N$, exactly one production rule from $a$ is in $P$. Thus, the language $L(G)$ defined by $G$ is a singleton set. We say a CFG $G$ derives $w \in \Sigma^{*}$ if $L(G)=\{w\}$. The size of $G$ is the total length of strings in the right hand sides of all production rules, and is denoted by $|G|$. The aim of grammar-based compression is formalized as a combinatorial optimization problem, as follows:

\section{Problem 1: Grammar-Based Compression}

InSTANCE: A string $w \in \Sigma^{*}$.

Solution: A deterministic CFG $G$ that derives $w$.

Measure: The size of $G$.

From now on, we assume that every deterministic CFG is in Chomsky normal form, i.e. the size of strings in the right-hand side of production rules is two, and we use $|N|$ for the size of a CFG. Note that for any CFG $G$, there is an equivalent CFG $G^{\prime}$ in Chomsky normal form such that $\left|G^{\prime}\right| \leq 2 \cdot|G|$

The approximation ratio of a grammar-based compression algorithm $A$ is defined by the quantity

$$
\max _{w \in \Sigma^{*}}\left\{\frac{\left|G_{A}(w)\right|}{\left|G_{o p t}(w)\right|}\right\},
$$

where $G_{A}(w)$ is the CFG computed by $A$ and $G_{o p t}(w)$ is an optimum CFG for a string $w$.

It is known that there is an important relation between a deterministic CFG and a factorization called $L Z$ factorization. The factorization for $w$, denoted by $L Z(w)$, is the decomposition of $w$ into $f_{1} \cdots f_{k}$, where $f_{1}=w[1]$, and for each $1<\ell \leq k, f_{\ell}$ is the longest prefix of the suffix $w\left[\left|f_{1} \cdots f_{\ell-1}\right|+1,|w|\right]$ that appears in $f_{1} \cdots f_{\ell-1}$, where $f_{\ell-1}$ is empty if $\ell=1$. Each $f_{\ell}$ is called a factor. The size $|L Z(w)|$ of $L Z(w)$ is the number of its factors. The following result is used in the analysis of the approximation ratio of our algorithm. 


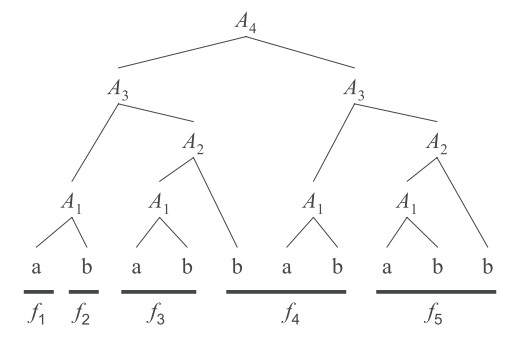

Fig. 1 LZ-factorization and CFG derivation.

Example 1: The relation of the size of LZ-factorization and CFG is illustrated in Fig. 1. For a string "ababbababb", the first two factors are $f_{1}=a$ and $f_{2}=b$. Similarly, we obtain the sequence

$$
f_{1}=a, f_{2}=b, f_{3}=a b, f_{4}=b a b, f_{5}=a b b .
$$

Figure 1 shows that the size of LZ-factorization is always smaller than or equal to that of any CFG. Note that the size of CFG is defined by $2|N|$.

Theorem 1 ([17]): For any string $w$ and its deterministic CFG $G$, the inequality $|L Z(w)| \leq|G|$ holds.

This theorem shows that the number of LZ factors is smaller than the size of a minimum CFG for any string.

\section{Compression by the Alphabetical Order}

In this section we describe the central idea of our grammarbased compression utilizing information only available from individual symbols. The aim is to minimize the number of different nonterminals generated by our algorithm.

A replacement $[i, i+1] \rightarrow$ a for $w$ is an operation that replaces a pair $w[i, i+1]$ with a nonterminal $a \in N$. A set $R$ of replacements is, by assuming some order on $R$, regarded as an operation that performs a series of replacements to $w$. In the following we introduce a definition of a set of replacements whose effect on a string is independent of the order.

Definition 1: A set $R$ of replacements for $w$ is appropriate if it satisfies the following: (1) At most one of two overlapping segments $[i, i+1]$ and $[i+1, i+2]$ is replaced by replacements in $R$, (2) At least one of three overlapping segments $[i, i+1],[i+1, i+2]$ and $[i+2, i+3]$ is replaced by replacements in $R$, and (3) For any pair of replacements $[i, i+1] \rightarrow a$ and $[j, j+1] \rightarrow b$ in $R, a=b$ if and only if $w[i, i+1]=w[j, j+1]$.

Clearly, for any string $w$, an appropriate replacement $R$ for $w$ generates the string $w^{\prime}$ uniquely. In such a case, we say that $R$ generates $w^{\prime}$ from $w$, and write $w^{\prime}=R(w)$. Intuitively, $w^{\prime}=R(w)$ is a resulting string by an execution of single loop of our compression algorithm, which continues the process till $\left|w^{\prime}\right|<|w|$.

Our first problem is to find small appropriate replacements, and here we explain the strategies for making pairs in our algorithm.

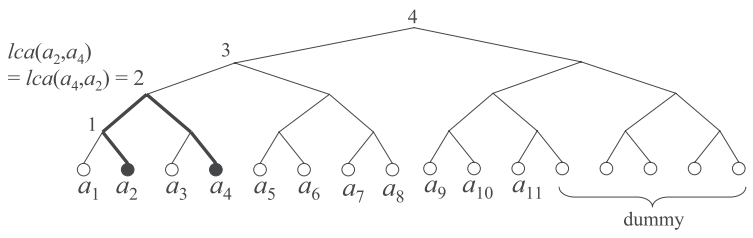

Fig. 2 The alphabet tree for $\Sigma \cup N=\left\{a_{1}, \ldots, a_{11}\right\}$.

Alphabet tree: Let $d$ be a positive integer, and let $k$ be $\left\lceil\log _{2} d\right\rceil$. An alphabet tree $T_{d}$ for $\Sigma \cup N=\left\{a_{1}, \ldots, a_{d}\right\}$ is the rooted, ordered complete binary tree whose leaves are labeled with $1, \ldots, 2^{k}$ from left to right. The height of an internal node refers to the number of edges of a path from the node to a descendant leaf. Let $h$ be the height of the lowest common ancestor of leaves $i$ and $j$. Then we define $l c a\left(a_{i}, a_{j}\right)_{d}=h$. Usually we omit the index $d$, and for the simplicity we assume that $l c a(i, j)$ is identical to $l c a\left(a_{i}, a_{j}\right)$. Moreover ' $\log$ ' denotes the binary logarithm throughout this paper.

Example 2: If $|\Sigma \cup N|=11$, the corresponding alphabet tree and the value of $l c a(i, j)$ are illustrated in Fig. 2.

For every string $w \in \Sigma^{+}$, any maximal repetition $w[i, j]=x^{k}$ is called type 1 metablock and any other occurrence of substring is called type 2 metablock of $w$. For example we illustrate the following factorization by type 1 and 2 metablock:

$$
w=a b c a b b c a a a b a b=a b c a \cdot b b \cdot c \cdot a a a \cdot b a b
$$

Any type 1 metablock $\alpha$ can be compressed to a sufficiently short string. For instance, if $\alpha=b^{2 k}$ for a symbol $b, \alpha$ is compressed to $A^{k}$ by $A \rightarrow b b$, and if $\alpha=b^{2 k+1}, \alpha$ is compressed to $A^{k} B$ by $A \rightarrow b b$ and $B \rightarrow b$. The trivial production rule $B \rightarrow b$ is produced to replace all symbols in the current string. This strategy is important to achieve our space-saving compression. In the next section, we introduce the general case of such compression called typical compression.

For type 2 metablocks, we introduced our iterative compression technique by $l c a$ and alphabet reduction.

Definition 2: Let $w$ be a type 2 metablock. $\quad w[i, i+1]$ is called to be maximal if $l c a(w[i], w[i+1])>l c a(w[i-$ $1], w[i]), l c a(w[i+1], w[i+2])$, where $w[1,2]$ is maximal if $l c a(w[1], w[2])>l c a(w[2], w[3])$, and the case $w[|w|-1,|w|]$ is similarly defined.

Our idea is to replace all occurrences of maximal pairs prior to others. Any two occurrences of maximal pairs are not overlapping, that is, if $w[i, i+1]$ is maximal, then neither $w[i-1, i]$ nor $w[i+1, i+2]$ is maximal. Thus, we can replace all the occurrences of maximal pairs by appropriate nonterminals. However there is a long substring $w[i, j]$ containing no maximal pair such that $|w[i, j]|=\lceil\log |\Sigma|\rceil$ in worst case. For instance, $a_{1} a_{2} a_{4} \cdots a_{2^{k}}$ is one of such strings. For improving such a bound, we compute $l c a(w[i], w[i+1])$ iteratively by the following strategy, which is a variant of alphabet reduction [5] defined on integers. We expand this notion 


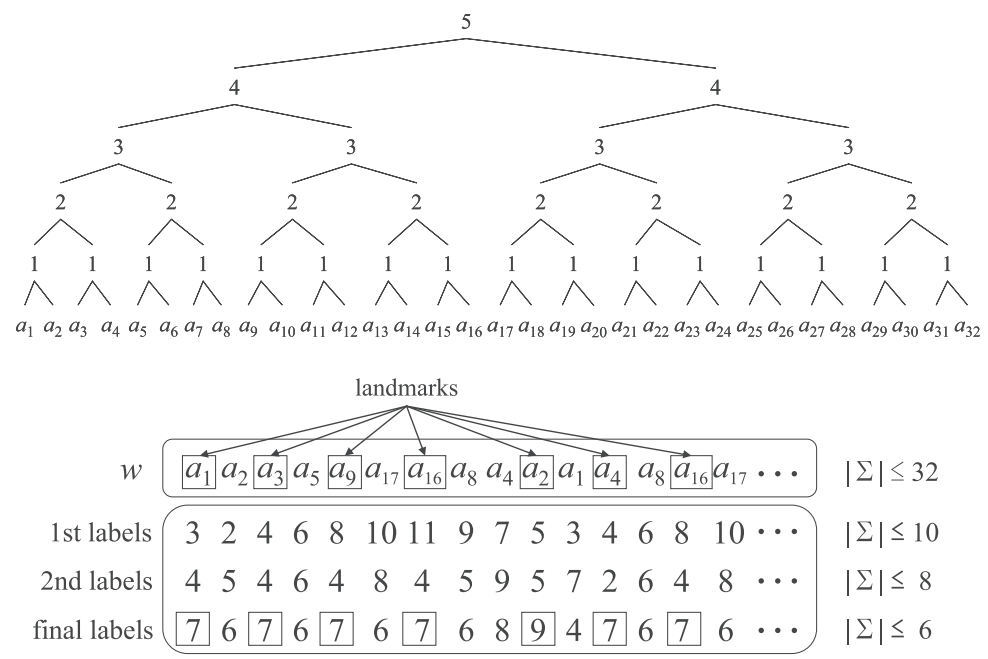

Fig. 3 A worst case $\log |\Sigma|$-iteration of alphabet reduction and resulting landmarks: each internal node in the tree denotes the value of $l c a$ for the corresponding leaves.

to alphabet trees for our compression problem.

Alphabet reduction: Let $w$ be a type 2 metablock. In case $k=2, \ldots,|w|$ and $w[k-1, k]=a_{i} a_{j}$, we define $\operatorname{label}(w[k])=2 \cdot \operatorname{lca}(i, j)$ if $i<j$ and $2 \cdot \operatorname{lca}(i, j)+1$ otherwise. In case $k=1$ and $w[1,2]=a_{i} a_{j}$, we define $\operatorname{label}(w[1])=2 \cdot \operatorname{lc} a(i, j)$ if $i>j$ and $2 \cdot \operatorname{lc} a(i, j)+1$ otherwise.

Lemma 1: For each $k$, if $w[k] \neq w[k+1]$, then $\operatorname{label}(w[k]) \neq \operatorname{label}(w[k+1])$.

proof. We show that $\operatorname{label}(w[\ell+1]) \neq \operatorname{label}(w[\ell+2])$ for $w[\ell, \ell+2]=a_{i} a_{j} a_{k}$. In case $(j>i, k)$ or $(j<i, k)$, exactly one of label $(w[\ell+1])$ and label $(w[\ell+2])$ is odd. In case $i<$ $j<k$, we obtain $l c a(i, j) \neq l c a(j, k)$. Moreover, label $(w[\ell+$ $1])=2 \cdot \operatorname{lca}(i, j)$ and $\operatorname{label}(w[\ell+2])=2 \cdot \operatorname{lca}(j, k)$ derives $\operatorname{label}(w[\ell+1]) \neq \operatorname{label}(w[\ell+2])$. The case of $i>j>k$ is similar.

Q.E.D.

From a string $w$ of length $n$, a sequence $w^{\prime}=$ label $(w[1])$ label $(w[2]) \cdots \operatorname{label}(w[n])$ is computed. By regarding each integer $\ell=\operatorname{label}(w[k])$ as a next alphabet symbol $a_{\ell}$, we then continue the alphabet reduction for the string $w$ iteratively. The purpose of the alphabet reduction is to reduce all symbols to constant integers preserving the structures of substrings. The next lemma shows that the number of iteration is very small.

Lemma 2: After at most $\log ^{*} n$ iterations of alphabet reduction, the label size is 6 .

proof. Let $w[k-1, k]=a_{i} a_{j}$ and $2 \leq k \leq n=|w|$. The size of the next label of $w[k]$ is reduced to label $(w[k]) \leq$ $\max \{2\lceil\log j\rceil, 2\lceil\log i\rceil\}+1$ by single iteration. Thus, the alphabet reduction terminates within $\log ^{*} n$ iterations. Moreover, at each iteration, the alphabet size goes from $|\Sigma|$ to at most $2\lceil|\log \Sigma|\rceil$. If $|\Sigma|>6$, then $2 \log \lceil|\Sigma|\rceil<|\Sigma|$, that is, the next label size is smaller than the current label size. Thus, the final labels are bounded by 6 .

Q.E.D.
If different symbols in $w$ are less than or equal to 6, the iteration of alphabet reduction terminates. When the iteration terminates for the string $w$, the resulting sequences label $(w[1])$ label $(w[2]) \cdots \operatorname{label}(w[n])$ is called a final labels, and a symbol $w[k]$ is called a landmark if label $(w[k])$ is maximal, i.e. label $(w[k])>\operatorname{label}(w[k-1]), \operatorname{label}(w[k+1])$, where $w[1]$ is maximal if $\operatorname{label}(w[1])>\operatorname{label}(w[2])$, and the case $w[|w|]$ is similar.

Here we note that any $w[i, j]$ in type 2 string longer than 6 must contain at least one landmark. Using this property, the aim of our algorithm is to synchronize the landmarks in all occurrences of a same substring.

Example 3: We show a worst case iteration of alphabet reduction in Fig. 3. In case that $|\Sigma| \leq 32$, if $w$ is formed by the string presented in Fig. $3, \log ^{*}|\Sigma|=3$ times iteration is necessary in worst case to obtain the finial label sequence.

\section{Algorithm and Analysis}

In this section we introduce an approximation algorithm for the grammar-based compression problem and analyze its approximation ratio to the optimum as well as its space efficiency.

\subsection{Algorithm LCA*}

Before the description of our algorithm, we first explain a typical compression for a trivial string. The following trivial replacement $R(w)=A_{1} \cdots A_{k}$ is called a typical compression for $w$ of length $n$.

$$
\begin{aligned}
& A_{1} \rightarrow w[1,2], A_{2} \rightarrow w[3,4], \ldots, \\
& \left\{\begin{array}{l}
A_{k} \rightarrow w[n-1, n], \text { if } n \text { is even } \\
A_{k-1} \rightarrow w[n-2, n-1], A_{k} \rightarrow w[n], \text { otherwise. }
\end{array}\right.
\end{aligned}
$$

The last replacement $A_{k} \rightarrow w[n]$ is called renaming. 


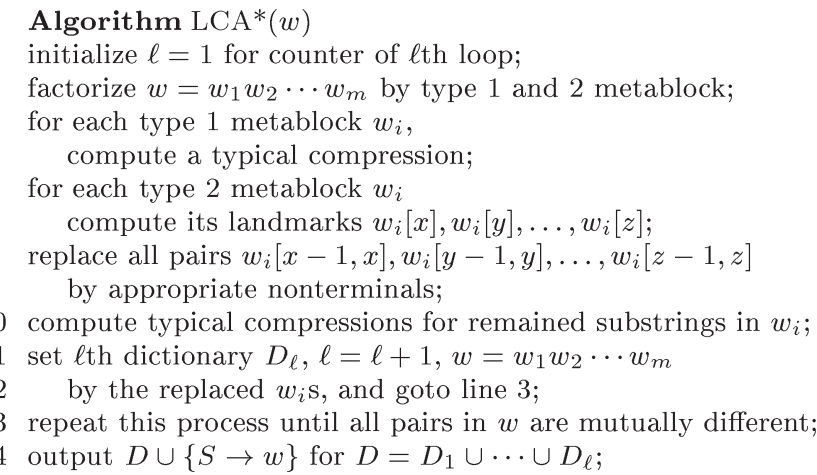

Fig. 4 The LCA* compression algorithm. A replaced pair $w[i, i+1]$ must be consistent with a current dictionary $D_{\ell}$, i.e. $w[i, i+1]$ is replaced by $A$ if a production $A \rightarrow B C(B C=w[i, i+1])$ is already registered to a $D_{\ell}$ and a new nonterminal is created to replace $w[i, i+1]$ otherwise.

In our compression algorithm, we assume any replacement is consistent to a current dictionary $D$, that is, any replaced pair $w[i, i+1]$ and $w[j, j+1]$ must be replaced by an identical nonterminal if $w[i, i+1]=w[j, j+1]$. The algorithm $\mathrm{LCA}^{*}(w)$ is presented in Fig. 4. We describe the outline of $\mathrm{LCA}^{*}(w)$ in Fig. 5 .

Phase 1 (Line 3):

The algorithm find all type 1 and type 2 metablocks in the input string $w$. Each metablock $w_{i}$ is compressed in Phase 2 and 3 individually.

Phase 2 (Line $4-5$ ):

Type 1 metablock substring $w_{i}$, i.e. a maximal repetition is replaced by a typical compression for $w_{i}$. If $\left|w_{i}\right|$ is odd, the last symbol is renamed; This trivial replacement is necessary for our space-saving compression. Such renaming is executed in the next phase.

Phase 3 (Line $6-10)$ :

Type 2 metablock $w_{i}$, i.e. $w_{i}[j] \neq w_{i}[j+1]$ for all $j$ is replaced. First, all landmarks in $w_{i}$ are found and for any landmark $w_{i}[j]$, the pair $w_{i}[j-1, j]$ is replaced by a nonterminal. Second, if $w_{i}[k]$ is the nearest landmark from $w_{i}[j]$, the remained substring $w_{i}[j+1, k-2]$ is replaced by a typical compression.

Phase 4 (Line $11-14)$ :

In $\ell$ th loop, let $D_{\ell_{1}}$ and $D_{\ell_{2}}$ be the set of production rules produced for type 1 and 2 metablocks, respectively. In this phase, the depth of loop $\ell$, the current string $w$, and the current dictionary $D$ are updated to $\ell=\ell+1, w=w_{1} w_{2} \cdots w_{m}$ by the compressed metablocks $w_{i}(1 \leq i \leq m)$, and $D=$ $D_{1} \cdots \cup D_{\ell}$. The above phases are repeated until all symbols

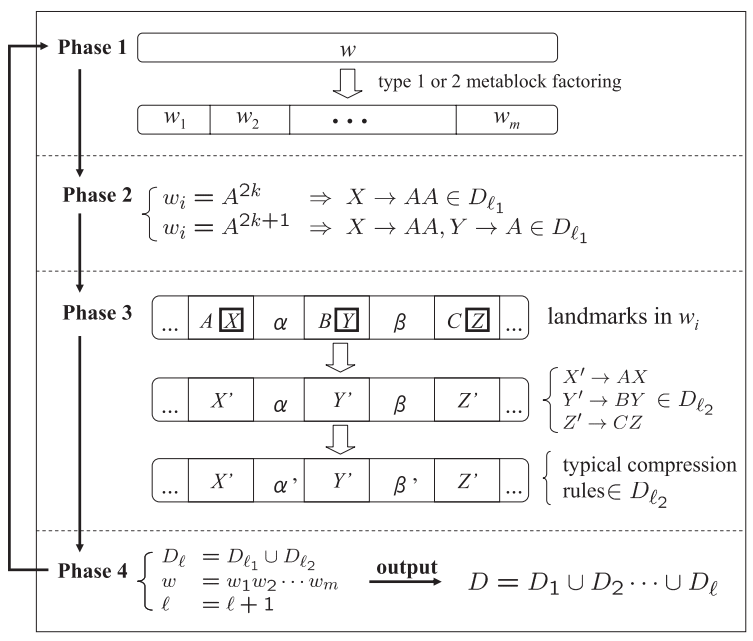

Fig. 5 The flow of LCA* for a string $w$.

are different in a current string. Then, the algorithm outputs the finial dictionary and terminates.

Theorem 2: The running time of $\operatorname{LCA}^{*}(w)$ is bounded by $O\left(n \log ^{*} n\right)$.

proof. By Lemma 2, the time to compute all landmarks is $O\left(\log ^{*} n\right)$ and it is clear that other computation is $O(n)$ time for each loop. Moreover, a current string shrinks in half approximately by single loop of LCA*. Thus, the total length of strings given to $\mathrm{LCA}^{*}$ is bounded by $O(n)$. Hence, the time complexity is $O\left(n \log ^{*} n\right)$.

Q.E.D.

\subsection{Performance Analysis}

Before the proof of our approximation ratio, we introduce a notion of occurrences of a substring.

Definition 3: For an occurrence $w[i, j]=\alpha$, we call it a boundary occurrence if $w[i-1] \neq w[i]$ and $w[j] \neq w[j+$ $1]$. In case $w[1, j]$, that is, a prefix of $w$, it is also called a boundary occurrence if $w[j] \neq w[j+1]$, and so is in suffixes.

Lemma 3: Let $\alpha$ be a substring in $w$ satisfying $\alpha=$ $w[\ell, r]=w\left[\ell^{\prime}, r^{\prime}\right]$. For any replacement by single loop of $\mathrm{LCA}^{*}(w)$, a pair in $w[\ell, r]$ is replaced iff the corresponding pair in $w\left[\ell^{\prime}, r^{\prime}\right]$ is replaced by a same nonterminal except at most $\log ^{*} n$ pairs in $w[\ell, r]$ and $w\left[\ell^{\prime}, r^{\prime}\right]$.

proof. By Lemma 1 and Lemma 2, for any type 2 metablock, we have following facts:

1. The final labels consist of at most 6 symbols and any label never repeats.

2. The final label of $w[i]$ depends on at most $\log ^{*} n$ symbols to its left.

If $w[\ell, r]$ and $w\left[\ell^{\prime}, r^{\prime}\right]$ are both boundary occurrences, there is a unique metablock factorization for them, like $\alpha=\alpha_{1} \cdots \alpha_{m}$. By the above facts, if $|\alpha|>2 \log ^{*} n, w[\ell, r]$ 
and $w\left[\ell^{\prime}, r^{\prime}\right]$ contain at least two landmarks in the same positions. Let $w[\ell+i]$ be such the left most landmark and $w[\ell+j]$ be the right most landmark. Thus, by the definition of replacement in our algorithm, the occurrences $w[\ell+i, \ell+j]$ and $w\left[\ell^{\prime}+i, \ell^{\prime}+j\right]$ are replaced identically.

If $w[\ell, r]$ is not boundary, the shortest boundary occurrence containing $w[\ell, r]$ is formed by $x^{+} \alpha_{1} \cdots \alpha_{m} y^{+}$for $x, y \in \Sigma$ and a metablock $\alpha_{i}$. In this case, the replacement of the boundary $\alpha_{1} \cdots \alpha_{m}$ in $w[\ell, r]$ and $w\left[\ell^{\prime}, r^{\prime}\right]$ are completely identical since all labels are decided within $\alpha_{1} \cdots \alpha_{m}$. Thus, in this case, disagreement of replacement occurs in the last symbol of $x^{+}$and $y^{+}$only.

Hence, in each case, the replacements of $w[\ell, r]$ and $w\left[\ell^{\prime}, r^{\prime}\right]$ for the same substring are identical except at most $\log ^{*} n$ pairs of them.

Q.E.D.

Theorem 3: The worst-case approximation ratio of the size of a grammar produced by the algorithm LCA* to the size of a minimum grammar is $O\left(\left(\log ^{*} n\right) \log n\right)$.

proof. Let $R$ be the set of appropriate replacements produced by single loop of $L C A^{*}(w)$. Let $g$ be the size of a minimum CFG for $w$, and let $w_{1} \cdots w_{k}$ be the $L Z$-factorization of $w$. We denote by $\#(w)_{R}$ the number of different nonterminals produced by $R$. From the definition of $L Z$-factorization, any factor $w_{i}$ occurs in $w_{1} \cdots w_{i-1}$, or $\left|w_{i}\right|=1$.

With lemma 3, any factor $w_{i}$ and its left-most occurrence are compressed into almost the same strings except $\log ^{*} n$ pairs in them. Thus, by the bound of the number of LZ-factors in Theorem [17], we can obtain the following estimation.

$$
\begin{aligned}
\#(w)_{R} & =\#\left(w_{1} \cdots w_{k-1}\right)_{R}+\log ^{*} n \\
& =\#\left(w_{1} \cdots w_{k-2}\right)_{R}+2 \log ^{*} n \\
& =O\left(k \log ^{*} n\right) \\
& =O\left(g \log ^{*} n\right)
\end{aligned}
$$

This is the number of different nonterminals produced by single loop execution in $\operatorname{LCA}^{*}(w)$. Clearly the loop is executed at most $O(\log n)$ times. Therefore, the total number of different nonterminals produced by $\operatorname{LCA}(w)$, that is, the size of CFG is $O\left(g\left(\log ^{*} n\right) \log n\right)$. This derives the approximation ratio.

Q.E.D.

The memory space required by $\mathrm{LCA}^{*}(w)$ can be bounded by the size of data structure to answer the membership query: input is a pair $A_{i} A_{j}$; output is an integer $k$ if $A_{k} \rightarrow A_{i} A_{j}$ is already created and no otherwise. By Theorem 3 , the size of a current dictionary $D_{\ell}$ is bounded by $O(g \log g)$ for each $\ell \geq 1$. Moreover, each symbol $A_{i}$ in a current string is replaced by a rule of the form $A_{j} \rightarrow A_{i}$ or $A_{j} \rightarrow Y Z$, where $A_{i} \in\{Y, Z\}$. Thus, $O\left((g \log g)^{2}\right)$-space algorithm is obtained by a naive implementation using look up table. Finally we show that the memory space can be improved to $O(g \log g)$.

\subsection{Improving the Space Efficiency}

An idea for improving space complexity of the algorithm is to recycle nonterminals created in the preceding iteration. Let $D(\alpha)$ be the string obtained by applying a dictionary $D$ to a string $\alpha$. Let $D_{1}$ and $D_{2}$ be dictionaries such that any symbol in $w$ is replaced by $D_{1}$ and any symbol in $D_{1}(w)$ is replaced by $D_{2}$. Then, the decoding of the string $D_{2}\left(D_{1}(w)\right)$ is uniquely determined, even if $D_{2}$ reuses nonterminals in $D_{1}$ like " $A \rightarrow A B$." Thus, we consider that the final dictionary $D$ is composed of $D_{1}, \ldots, D_{\ell}$, where $D_{i}$ is the dictionary constructed in the $i$ th iteration. Since all symbols in $w$ are replaced within a same loop in $\mathrm{LCA}^{*}$, the decoding from the final string $w^{\prime}$ is uniquely decided by the semantics $D_{m}\left(\cdots D_{1}\left(w^{\prime}\right) \cdots\right)=w$. Such a dictionary is computed by the following function and data structures. Let $D_{i}$ be the set of productions, $N_{i}$ the set of alphabet symbols created in the $i$ th iteration, and $k_{i}$ the cardinality $\left|N_{i}\right|$. We define the function

$$
f_{i}(x, y)=(x-1) k_{i}+y \text { for } x, y=1, \ldots, k_{i} .
$$

This is a one-to-one mapping from $\left\{1, \ldots, k_{i}\right\} \times\left\{1, \ldots, k_{i}\right\}$ to $\left\{1, \ldots, k_{i}^{2}\right\}$, and is used to decide an index of a new nonterminal associated to a pair $A_{x} A_{y}$, where $A_{x}$ denotes the $x$ th created nonterminal in $N_{i}$.

The next dictionary $D_{i+1}$ is constructed from $D_{i}, N_{i}$, and $f_{i}$ as follows. In the algorithm $\mathrm{LCA}^{*}$, there are two cases of replacements: one is for replacements of pairs, and the other is renaming. We first explain the case of replacements of pairs. Let a pair $A_{x} A_{y}$ in a current string be decided to be replaced. The algorithm LCA* computes the integer $z=f(x, y)$, and looks up a hash table $H$ for $z$. If $H(z)$ is absent and $N_{i}=\left\{A_{1}, \ldots, A_{k}\right\}$, then set $N_{i}=N_{i} \cup\left\{A_{k+1}\right\}$, $D_{i}=D_{i} \cup\left\{A_{k+1} \rightarrow A_{x} A_{y}\right\}, H(z)=k+1$, and replace the pair $A_{x} A_{y}$ with $A_{k+1}$. If $H(z)=k+1$ is present, then only replace the pair $A_{x} A_{y}$ by $A_{k+1}$. For the case of renaming of a symbol $A_{x}$, we can use the nonterminal $A_{k+1}$ such that $z=f_{i}(x, x)$ and $H(z)=k+1$. The dictionary $D_{i}$ constructed in the $i$ th iteration can be divided to $D_{i_{1}}$ and $D_{i_{2}}$ such that $D_{i_{1}}$ is the dictionary for repetitions and $D_{i_{2}}=D_{i} \backslash D_{i_{1}}$. Thus, we can create all productions without collisions, and decode a current string $w_{i+1}$ to the previous string $w_{i}$ by the manner $D_{i}\left(w_{i+1}\right) \equiv D_{i_{1}}\left(D_{i_{2}}\left(w_{i+1}\right)\right)=w_{i}$.

Theorem 4: The space required by $\mathrm{LCA}^{*}(w)$ is $O(g \log g)$ for the size of a minimum CFG for $w$.

proof. Let $n=|w|$ and $\ell$ be the number of iterations of loops executed in $\operatorname{LCA}^{*}(w)$. By theorem 3 , the number $\left|N_{i}\right|$ of new nonterminals created in the $i$ th iteration is $O\left(g \log ^{*} n\right)$ for each $i \leq \ell$. To decide the index of a new nonterminal from a pair $A_{x} A_{y}, \mathrm{LCA}^{*}$ computes $z=f_{i}(x, y), H(z)$, and $k=\left|N_{i}\right|$ for the current $N_{i}$. Since $|z| \leq O(\log n)$ and the number of different $z$ is $O(g \log g)$, the space for $H$ is $O(g \log g)$ and $k=O(g \log g)$. Thus, the construction of $D_{i}$ requires only $O(g \log g)$ space. We can release whole the memory space for $D_{i}$ in the next loop. Hence, the total space for constructing $D$ is also $O(g \log g)$.

Q.E.D. 
Table 1 Performance of compression algorithms: the common lower bound $\Omega(n)$ for the time complexity is omitted, ' $\alpha$ ' and '-' denote $\log ^{*} n$ and unknown, respectively, and Rytter [17], Welch [21] contains LZ77, LZ78 algorithms by Ziv and Lempel [23], [24], respectively.

\begin{tabular}{|c|c|c|c|}
\hline $\begin{array}{c}\text { Algorithm } \\
\text { [reference] }\end{array}$ & $\begin{array}{c}\text { Approx. Ratio } \\
\text { upper/lower }\end{array}$ & $\begin{array}{c}\text { Space } \\
\text { upper/lower }\end{array}$ & $\begin{array}{c}\text { Time } \\
\text { upper }\end{array}$ \\
\hline proposed & $\alpha \log n /-$ & $g \log g / \log n$ & $\alpha n$ \\
Charikar [4] & $\log n /-$ & $n / n$ & $n$ \\
Rytter [17] & $\log n /-$ & $n / n$ & $n$ \\
Sakamoto [19] & $\log n /-$ & $n / n$ & $n$ \\
Welch [21] & $\left(\frac{n}{\log n}\right)^{\frac{2}{3}} / \sqrt[3]{\log n}$ & $n / n$ & $n$ \\
Larsson [10] & $\left(\frac{n}{\log n}\right)^{\frac{2}{3}} / \sqrt{\log n}$ & $n / n$ & $n$ \\
Witten [16] & $\left(\frac{n}{\log n}\right)^{\frac{3}{4}} / \sqrt[3]{n}$ & $n / n$ & $n$ \\
Kieffer [7] & - & $n / n$ & $n$ \\
Kieffer [8] & $\left(\frac{n}{\log n}\right)^{\frac{1}{2}} / \frac{\sqrt{n}}{\log n}$ & $\sqrt{n \log n} / \sqrt{n}$ & $n$ \\
& $\left(\frac{n}{\log n}\right)^{\frac{2}{3}} / 1.37$ & $n \log n / n$ & $n \log ^{2} n$ \\
Apostolico [3] & & \\
\hline
\end{tabular}

\subsection{Comparison with Related Works}

In Table 1, we summarize all the results obtained in this study together with related works on grammar-based compression, where the trivial time complexity $\Omega(n)$ is omitted. In this table, all results concerned with approximation ratio were proved in [12] as well as the upper/lower bounds of the grammar size by each algorithm, which directly derives the complexity bounds. In particular, the reason for $\Omega(n)$ space of almost algorithms is due to the data structures of indexing for substrings. Since our algorithm does not require no index for substring, the required space depends on the hash table, that is, the grammar size only.

\section{Conclusion}

We presented a space-efficient near linear-time algorithm for the smallest CFG problem. This algorithm guarantees the approximation ratio $O\left(\left(\log ^{*} n\right) \log n\right)$, which is almost $O(\log n)$ and the memory space $O(g \log g)$ for the minimum CFG size $g$ of input string. This space bound is considered to be sufficiently small since $\Omega(g)$ space is a lower bound for non-adaptive dictionary-based compression. In addition, it is known that $\Omega(\log n) \leq g \leq O\left(\frac{n}{\log _{k} n}\right)$ [12] for $k=|\Sigma|$. The upper bound of memory space is best in the previously known polylog-approximation algorithms. Practically, production rules for renaming occupy comparatively large space in final dictionary. However it is still open whether it is possible to reduce such renaming production rules preserving the time/space complexity.

\section{Acknowledgments}

This research was partially supported by the Ministry of Education, Science, Sports and Culture, Grant-in-Aid for Young Scientists (B), 18700154, 2006-2007, and Scientific Research (B), 19300008, 2007-2008. The authors would like to thank the anonymous referees of SPIRE2004, ISAAC2006, and IEICE Transactions on Information and Systems for the valuable comments to revise the preliminary version of this paper.

\section{References}

[1] G. Ausiello, P. Crescenzi, G. Gambosi, V. Kann, A. MarchettiSpaccamela, and M. Protasi, Complexity and Approximation: Combinatorial Optimization Problems and Their Approximability Properties, Springer, 1999.

[2] S. De Agostino and J.A. Storer, "On-line versus off-line computation in dynamic text compression," Inf. Process. Lett., vol.59, pp.169174, 1996.

[3] A. Apostolico and S. Lonardi, "Some theory and practice of greedy off-line textual substitution," Proc. Data Compression Conference 1998, pp.119-128, 1998.

[4] M. Charikar, E. Lehman, D. Liu, R. Panigrahy, M. Prabhakaran, A. Rasala, A. Sahai, and A. Shelat, "The smallest grammar problem," IEEE Trans. Inf. Theory, vol.51, no.7, pp.2554-2576, 2005.

[5] G. Cormode and S. Muthukrishnan, "The string edit distance matching problem with moves," ACM Trans. Algorithms, vol.3, no.1, article 2, 2007

[6] T. Kida, T. Matsumoto, Y. Shibata, M. Takeda, A. Shinohara, and S. Arikawa, "Collage system: A unifying framework for compressed pattern matching," Theor. Comput. Sci., vol.298, no.1, pp.253-272, 2003.

[7] J.C. Kieffer and E.-H. Yang, "Grammar-based codes: A new class of universal lossless source codes," IEEE Trans. Inf. Theory, vol.46, no.3, pp.737-754, 2000.

[8] J.C. Kieffer, E.-H. Yang, G. Nelson, and P. Cosman, "Universal lossless compression via multilevel pattern matching," IEEE Trans. Inf. Theory, vol.46, no.5, pp.1227-1245, 2000.

[9] D. Knuth, The Art of Computer Programming, Volume II: Seminumerical Algorithms, Addison-Wesley, 1981.

[10] N.J. Larsson and A. Moffat, "Offline dictionary-based compression," Proc. IEEE, vol.88, no.11, pp.1722-1732, 2000.

[11] J. van Leeuwen, (Managing Editor). Handbook of Theoretical Computer Science, Volume A: Algorithms and Complexity, Elsevier, 1998.

[12] E. Lehman, Approximation Algorithms for Grammar-Based Compression. PhD thesis, MIT, 2002.

[13] E. Lehman and A. Shelat, "Approximation algorithms for grammarbased compression," Proc. 20th Ann. ACM-SIAM Sympo. Discrete Algorithms, pp.205-212, 2002.

[14] S. Maruyama, H. Miyagawa, and H. Sakamoto, "Improving time and space complexity for compressed pattern matching," Proc. 17th Int. Sympo. Algorithms and Computation, LNCS4288, pp.484-493, 2006.

[15] C. Nevill-Manning and I. Witten, "Compression and explanation using hierarchical grammars," Comput. J., vol.40, no.2/3, pp.103-116, 1997.

[16] C. Nevill-Manning and I. Witten, "Identifying hierarchical structure in sequences: A linear-time algorithm," J. Artificial Intelligence Research, vol.7, pp.67-82, 1997.

[17] W. Rytter, "Application of Lempel-Ziv factorization to the approximation of grammar-based compression," Theor. Comput. Sci., vol.302, no.1-3, pp.211-222, 2003. 
[18] H. Sakamoto, T. Kida, and S. Shimozono, "A space-saving lineartime algorithm for grammar-based compression," Proc. 11th International Symposium on String Processing and Information Retrieval, LNCS3109, pp.218-229, 2004.

[19] H. Sakamoto, "A fully linear-time approximation algorithm for grammar-based compression," J. Discrete Algorithms, vol.3, no.24, pp.416-430, 2005.

[20] J.A. Storer and T.G. Szymanski, "The macro model for data compression," Proc. 10th Ann. Sympo. Theory of Computing, pp.30-39, 1978.

[21] T.A. Welch, "A technique for high performance data compression," Computer, vol.17, pp.8-19, 1984.

[22] E.-H. Yang and J.C. Kieffer, "Efficient universal lossless data compression algorithms based on a greedy sequential grammar transform-part one: Without context models," IEEE Trans. Inf. Theory, vol.46, no.3, pp.755-777, 2000.

[23] J. Ziv and A. Lempel, "A universal algorithm for sequential data compression," IEEE Trans. Inf. Theory, vol.IT-23, no.3, pp.337$349,1977$.

[24] J. Ziv and A. Lempel, "Compression of individual sequences via variable-rate coding," IEEE Trans. Inf. Theory, vol.IT-24, no.5, pp.530-536, 1978.

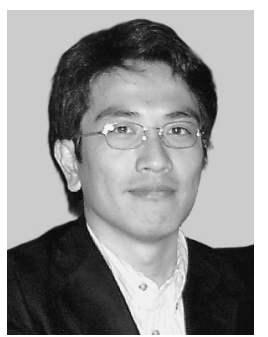

Hiroshi Sakamoto received the B.S. degree in Physics, the M.S. and Dr. Sci. degree in Information Systems all from Kyushu University in 1994, 1996, and 1998, respectively. He received JSPS Research Fellowships for Young Scientists from 1996 to 1998. From Jan. 1999 to Oct. 2003, he was a research associate of Department of Informatics, Kyushu University. He is currently an associate professor of Graduate School of Computer Science and Systems Engineering, Kyushu Institute of Technology. His research interests include algorithms in data compression and web mining. $\mathrm{He}$ is a member of JSAI and DBSJ.

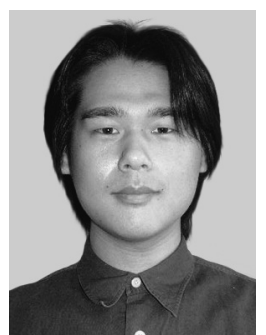

Shirou Maruyama received the B.S. degree in Engineering from Fukuoka University in 2006, and the M.S. degree in Computer Science and Systems Engineering from Kyushu Institute of Technology in 2008. He is currently a doctoral student in Department of Informatics, Kyushu University. His research interests include data compression and string pattern matching algorithms.

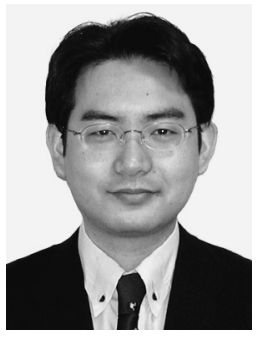

Takuya Kida received the B.S. degree in Physics, the M.S. and Dr.(Information Science) degree all from Kyushu University in 1997, 1999, and 2001, respectively. He was a Full-time Lecturer of Kyushu University Library from October 2001 to March 2004. He is currently an Associate Professor of Division of Computer Science, Graduate School of Information Science and Technology, Hokkaido University, since April 2004. His research interests include Text Algorithms and Data Structures, Information Retrieval, and Data compression. He is a member of JSPS and DBSJ.

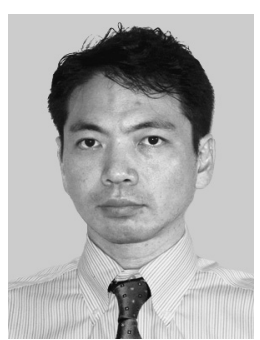

Shinichi Shimozono received $\mathrm{Ph}$. D. in Science from Graduate School of Information Science in Kyushu University in 1996. From 1992, he was a research associate at Kyushu Institute of Technology, and since 1996, he is working as an associate professor of Theoretical Computer Science branch at Department of Artificial Intelligence, Kyushu Institute of Technology. His research interest includes design and analysis of algorithms for intractable and brand-new computation problems especially for combinatorial optimization problems that are NP-hard, and analysis of computational hardness of combinatorial problems. 